\title{
Performance of EuroSCORE II in Predicting Early Mortality after Mitral, Aortic or Mitral \& Aortic Valve Surgery Patients in National Heart Foundation Hospital and Research Institute
}

Ahsan Uddin Mahmud ${ }^{1}$, Md Faizus Sazzad ${ }^{2}$, Mohammed Armane Wadud ${ }^{2}$, Iftakher Raja Chowdhury ${ }^{3}$, Mohammad Hafizur Rahman ${ }^{3}$, Haroon Rasheed Chowdhury ${ }^{3}$, Noel Cipriyan Gomes ${ }^{3}$, Sohel Reza Choudhury ${ }^{3}$, Quamrul Islam Talukdar $^{3}$, Prasanta Kumar Chanda ${ }^{3}$, Farooque Ahmed ${ }^{4}$

\begin{abstract}
:
Background: Preoperative risk assessment before cardiac surgery to predict mortality become literally important and practicing worldwide, whereas EuroSCORE II is most updated and popular. So we examined the hypothesis that Performance of EuroSCORE II in predicting early mortality after Mitral, Aortic or mitral \& aortic valve surgery patients in National Heart Foundation Hospital and Research Institute.

Objectives: To compare Euro SCORE II predicted early mortality and observed early mortality in a sample of patients of National Heart Foundation Hospital who underwent for Mitral, Aortic or Mitral \& Aortic valve surgery.

Methods: An observational prospective study was done in Department of cardiac surgery, National Heart Foundation Hospital and Research Institute who underwent for Mitral, Aortic or Mitral \& Aortic valve surgery in the period of July 2016 to March 2018. Sample size was $\mathbf{3 5 6}$ and all inclusion criteria full filled. Patients were divided into 3 group (low, medium \& high)
\end{abstract}

depending on the score. Model discrimination and calibration were assessed additive and logistic EuroSCORE and EuroSCORE II.

Results: The in hospital mortality of this series was $2.8 \%$ (10 out of 356 ) and the predicted mortality was $2.73 \%$ (95\% Cl 1.02-4.38) by the EuroSCORE II, 2.15\% (95\% Cl 0.68$3.72)$ by the additive method and $2.25 \%(95 \% \mathrm{Cl} 0.74-3.86)$ by the logistic EuroSCORE. The model's discriminatory power also good and useful as indicated by an area under ROC curve of 0.779 in EuroSCORE II model, 0.675 in additive method and 0.696 in logistic method that means EuroSCORE II method can predict the outcome with $77 \%$ accuracy, additive method with $67 \%$ accuracy and the logistic method does that with $69 \%$ accuracy.

Conclusion: EuroSCORE II was validated and performed well on National Heart Foundation patients and could be recommended as a simple risk stratification system to estimate the probability of early mortality in patients scheduled for valve surgery in Bangladesh.

Key Words: EUROSCORE-II, Heart Valve Surgery

1. Registrar, Department of Cardiac Surgery, Chittagong Medical College Hospital, Chattagram, Bangladesh.

2. Assistant Professor \& Associate Consultant, Department of Cardiac Surgery, Ibrahim Cardiac Hospital \& Research Institute, Dhaka, Bangladesh.

3. Cardiac Surgeon, Department of Cardiac Surgery, National Heart Foundation Hospital \& Research Institute, Dhaka, Bangladesh.

4. Professor \& Chief Cardiac Surgeon, Department of Cardiac Surgery, National Heart Foundation Hospital \& Research Institute, Dhaka, Bangladesh.

Address of Correspondence: Dr Md Faizus Sazzad, MBBS, MS, FCPS, FACS, Assistant Professor \& Associate Consultant, Department of Cardiac Surgery, Ibrahim Cardiac Hospital \& Research Institute, Dhaka, Bangladesh. Tel: +8801727210741, Email: mfsazzad@gmail.com 


\section{Introduction}

Preoperative risk stratification has become a core issue for patients with valve surgery, because progress in operative and perioperative care has led to a wide extension of surgical indications, addressing by surgical intervention even patients with severe multiple comorbidities who were previously treated with medical therapy. In this scenario, perioperative death cannot be considered the only quality measure, and the overall preoperative assessment of patients should also take into account the life expectancy after the valve operation (Fabio, et al., 2016).

EuroSCORE (European System for Cardiac Operative Risk Evaluation) is a risk model which allows the calculation of the risk of death after a heart operation. EuroSCORE, first developed as an additive model (additive EuroSCORE, AES) first published in 1999. It was based on data collected from 128 centers of eight European countries (German, France, United Kingdom, Italy, Spain, Finland, Sweden and Switzerland). The model asks for 17 items of information about the patient, the state of the heart and the proposed operation, and uses logistic regression to calculate the risk of death. However this model generally overestimates mortality in low risk patients and underestimates it in high risk groups (Shanmugam,et al., 2005).

Then logistic EuroSCORE (LES) was published in 2003 (Roques, et al., 2003) to gain predictive performance in high risk group. The model has been adopted worldwide, becoming the most widely used risk index for cardiac surgery. Over the last few years, several professionals from many parts of the world have reported that the model now overpredicts risk, as a result of cardiac surgery have improved significantly. To overcome this effect an updated version of this model named EuroSCORE II - was announced at the EACTS meeting in Lisbon on Monday, 3 October 2011 and published in the European Journal of Cardiothoracic Surgery in April 2012, based on the logistic regression analysis of 23,000 patients in 150 hospitals in 43 countries over a 12 week period (May July 2010) (Mohammad, et al., 2016).

Euroscore II, the updated version, has better discriminative power \& calibration. The discriminative power is important to determine how the model distinguishes between alive \& died patients during in-hospital period .Calibration is also important to determine the agreement between the real observed \& predicted mortality. The discriminatory power and precision in risk prediction of the EuroSCORE -II in mitral and aortic valve surgery has recently become increasingly important for 2 reason .First - In the most centers, valve procedures, either isolated or combined actually represent more than $30 \%$ of the total caseload, therefore, accurate risk estimation in this patient population, mainly elderly and very elderly people has become much more important. The second reason, is strictly related to the recent evolution in technical options in valve operations- minimally invasive valve surgery in patients at the high risk.

In UK, a dedicated website collected prospective risk and outcome data on 22,381 consecutive patients undergoing major cardiac surgery in 154 hospitals in 43 countries over a 12-week period (May-July 2010). Information was obtained on existing EuroSCORE risk factors and additional factors proven to influence risk from research conducted since the original model. The outcome was early mortality after valve surgery (within 30 days). A logistic risk model (EuroSCORE II) was then constructed and tested. Results shows Compared with the original 1995 EuroSCORE database (in brackets) Overall mortality was $3.9 \%(4.6 \%)$. When applied to the current data, the old risk models over predicted mortality (actual: 3.9\%; additive predicted: 5.8\%; logistic predicted: $7.57 \%$ ). EuroSCORE II was well calibrated on testing in the validation data subset of 5553 patients (actual mortality: 4.18\%; predicted: $3.95 \%$ ). Very good discrimination was maintained with an area under the receiver operating characteristic curve of 0.8095 (Nashef, et al., 2012).

A literature search identified 37 potentially eligible studies, and 12 were selected for meta-analysis comprising 26,621 patients with 1250 events (mortality rate, $4.7 \%$ ). Meta-analysis of these studies provided an average area under the curve (AUC) value of 0.730 . The same results were obtained when meta-analyses were performed separately, in the seven studies reporting (8175 patients with 358 events; mortality rate, $4.4 \%$ ), the ROC curve provided an average AUC value of 0.724 . The five studies not reporting reliable uncertainty estimation $(18,446$ patients with 892 events; mortality rate, 4.8\%) had an average AUC of 0.732. Studies documented a constant trend to over predict mortality by EuroSCORE, both in the additive and especially in the logistic form. In conclusion, The EuroSCORE has low discrimination ability for valve surgery, and it sensibly over predicts risk. Alternative risk scoring algorithms, EuroSCORE II should be seriously considered (Alessandro, et al., 2010).

National Heart Foundation Hospital and Research Institute (NHFH\&RI), Dhaka, is a high volume cardiac center in Bangladesh. At NHFH\&RI, previously a study was done to assess the performance of former EuroSCORE model among coronary artery bypass grafting (CABG) patients (Rahman MZ, et al.2012) although performance of EuroSCORE II has never been tested in valve surgery patients. The aim of this work in this center is to evaluate the current clinical profile of patients submitted to mitral and aortic valve surgery and 
to check expected risk of death in this group of patients by applying EuroSCORE II and compare its predictive performance with additive EuroSCORE (AES) and logistic EuroSCORE (LES) in terms of calibration and discrimination, thus to see whether EuroSCORE II could validate risks on our patient at NHFH\&RI.

Rationale: The European System for Cardiac Operative Risk Evaluation II (EuroSCORE II) is an updated version of the original EuroSCORE and used for prediction of early mortality in cardiac surgery patients in Europe. This risk scoring system has been found highly successful on CABG patients and previously two study were conducted at NHFH\&RI. But its validity was never been tested on mitral and aortic valve surgery of our population.

At present, NHFH\&RI a high volume center in Bangladesh operating various valve surgery which is about more than $30 \%$ of total surgery. The EuroSCORE II predicted value can be used in mitral and aortic valvular surgery for preoperative decision making \& risk assessment, counselling of patient, fitness of patients ,management planning, postoperative outcome \& follow-up and make a comparison between institutions and surgeons.

So, significant differences in the demography, risk profiles, surgical strategy in different countries of the world made individual population study necessary to confirm the predictive value of this pan-European method in that population.

The purpose of this study is to see whether EuroSCORE II can predict early mortality after elective mitral and aortic valve surgery \& optimize its usefulness in valve surgery in NHFH\&RI and compare its predictive performance with additive and logistic EuroSCORE.

\section{Materials \& Methods}

Study design: Prospective observational Study.

Place of study: Department of Cardiac surgery, National Heart Foundation Hospital \& Research Institute, Mirpur, Dhaka.

\section{Period of study: July 2016 to March 2018}

Ethical Issue: Permission was taken from the academic and institutional ethical and review board (IRB) of National Heart Foundation Hospital and Research Institute for conducting the study.

Study population: Patients operated in the department of cardiac surgery at NHFH \& RI, underwent Mitral, Aortic or mitral \& aortic valve surgery under CPB irrespective of sex.

Sampling Techniques: Purposive sampling.

\section{Selection criteria:}

a. Inclusion Criteria:

Adult Patients (>18 years) who will plan for Mitral, Aortic or mitral \& aortic valve surgeries at National Heart Foundation Hospital, Dhaka. b. Exclusion Criteria:

Patients planned for cardiac surgery other than mitral and aortic valve surgery (congenital heart disease, scheduled insertion of a cardiac assist device, operation on the aorta, thrombo-embolectomy of the pulmonary arteries, CABG, emergency procedure)

Patients undergoing redo-surgery

\section{Grouping of the patients:}

Patients will be prospectively allocated into three groups according to EuroSCORE II:

Low risk group (EuroSCORE II 0-2)

Medium risk group (EuroSCORE II 2-5)

High risk group (EuroSCORE II >5)

Measures of Variables:

EuroSCORE II Preoperative variables:

a. Patient related factors: Age, Gender, Creatinine clearance (normal $>85 \mathrm{ml} / \mathrm{min}$ ), Extra cardiac arteriopathy, Poor mobility, Previous cardiac surgery, Chronic lung disease, Active endocarditis, Critical preoperative state, Diabetes on insulin

b. Cardiac related factors: NYHA functional class, CCS class IV angina, LV dysfunction, Recent MI, Pulmonary hypertension

c. Operation related factors: Urgency (Elective/Urgent/ Emergency/Salvage), Weight of the intervention, Surgery on thoracic aorta

\section{Variables of additive and logistic EuroSCORE:}

A Patient related factors: Age, Female Gender, Chronic pulmonary disease, Extracardiac arteriopathy, Neurological dysfunction affecting mobility, Previous cardiac surgery, Bronchodilators or steroids for lung disease, Active endocarditis, Critical preoperative state

B. Cardiac related factors: Unstable angina, LV dysfunction, Recent myocardial infarction, Pulmonary hypertension

C. Operation related factors: Urgency (Elective/Urgent/ Emergency/Salvage), Other than isolated surgery, Post infarct septal rupture, Surgery on thoracic aorta

\section{Predicted variables}

1) Predicted mortality according to EuroSCORE II

2) Predicted mortality according to Additive EuroSCORE

3) Predicted mortality according to Logistic EuroSCORE

Post-operative variable:

Observed early mortality-

Died within 30 days from operation or later than 30 days if still in hospital. 
Weights (score) of additive and logistic EuroSCORE:

Patient related factors

\begin{tabular}{|c|c|c|c|}
\hline Risk factors & Definition & Additive score & Logistic score \\
\hline Age & Continues ( per 5 years of part thereof over 60 years) & 1 & 0.0666354 \\
\hline Sex & Female & 1 & 0.3304052 \\
\hline $\begin{array}{l}\text { Chronic pulmonary } \\
\text { disease }\end{array}$ & $\begin{array}{l}\text { Long term use of bronchodilators or steroids for } \\
\text { lung disease }\end{array}$ & 1 & 0.4931341 \\
\hline Extracardiac arteriopathy & $\begin{array}{l}\text { any one or more of the following: claudication, } \\
\text { carotid occlusion or }>50 \% \text { stenosis, previous or } \\
\text { planned intervention on the abdominal aorta, limb } \\
\text { arteries or carotids }\end{array}$ & 2 & 0.6558917 \\
\hline $\begin{array}{l}\text { Neurological dysfunction } \\
\text { disease }\end{array}$ & $\begin{array}{l}\text { severely affecting ambulation or day-to-day } \\
\text { functioning }\end{array}$ & 2 & 0.841626 \\
\hline Previous cardiac surgery & requiring opening of the pericardium & 3 & 1.002625 \\
\hline Active endocarditis & $\begin{array}{l}\text { patient still under antibiotic treatment for } \\
\text { endocarditis at the time of surgery }\end{array}$ & 3 & 1.101265 \\
\hline Critical preoperative state & $\begin{array}{l}\text { Any one or more of the following: ventricular } \\
\text { tachycardia or fibrillation or aborted sudden death, } \\
\text { preoperative cardiac massage, preoperative } \\
\text { ventilation before arrival in the anesthetic room, } \\
\text { preoperative inotropic support, intraaortic balloon } \\
\text { counter pulsation or preoperative acute renal } \\
\text { failure (anuria or oliguria< } 10 \mathrm{ml} / \mathrm{hour} \text { ) }\end{array}$ & 3 & 0.9058132 \\
\hline Serum creatinine & >200 micromole/l preoperatively & 2 & 0.6521653 \\
\hline
\end{tabular}

Cardiac related factors:

\begin{tabular}{llcc}
\hline Risk factors & Definition & Additive score & Logistic score \\
\hline Unstable angina & resting angina requiring iv nitrates until & 2 & 0.5677075 \\
& arrival in the anesthetic room & & \\
LV dysfunction & moderate or LVEF30-50\% & 1 & 0.4191643 \\
& poor or LVEF $<30$ & 3 & 1.094443 \\
Recent & $(<90$ days $)$ & 2 & 0.5460218 \\
$\begin{array}{l}\text { myocardial } \\
\text { infarct }\end{array}$ & & & \\
$\begin{array}{l}\text { Pulmonary } \\
\text { hypertension }\end{array}$ & Systolic PA pressure $>60 \mathrm{mmHg}$ & 2 & 0.7676924 \\
\hline
\end{tabular}

\section{Operation related factors:}

\begin{tabular}{|c|c|c|c|}
\hline Risk factors & Definition & Additive score & Logistic score \\
\hline $\begin{array}{l}\text { Emergency } \\
\text { operation }\end{array}$ & $\begin{array}{l}\text { Carried out on referral before the } \\
\text { beginning of the next working day }\end{array}$ & 2 & 0.7127953 \\
\hline $\begin{array}{l}\text { Other than } \\
\text { isolated CABG }\end{array}$ & $\begin{array}{l}\text { Major cardiac procedure other than or in } \\
\text { addition to CABG }\end{array}$ & 2 & 0.5420364 \\
\hline $\begin{array}{l}\text { Surgery on } \\
\text { thoracic aorta }\end{array}$ & For disorder of ascending aorta & 3 & 1.159787 \\
\hline $\begin{array}{l}\text { Post- infarct } \\
\text { septal rupture }\end{array}$ & & 4 & 10462009 \\
\hline
\end{tabular}


Beta co-efficient value of factors of EuroSCORE II:

Patient related factors

\begin{tabular}{lc}
\hline Risk factors & Beta co-efficient value for EuroSCORE II \\
\hline Age & 0.0285181 \\
Female sex & 0.02196434 \\
Chronic pulmonary disease & 0.1886564 \\
Extracardiac arteriopathy & 0.5360568 \\
Neurological dysfunction disease & 0.2407181 \\
Previous cardiac surgery & 1.118599 \\
Active endocarditis & 0.6194522 \\
Critical preoperative state & 1.086517 \\
Insulin dependant diabetes mellitus & 0.3542749 \\
\hline
\end{tabular}

For age, $\mathrm{Xi}=1$ if patient age $<60$; $\mathrm{Xi}$ increases by one point per year thereafter (age 60 or less $\mathrm{Xi}=1$; age 61 if $\mathrm{Xi}=2$; age 63 if $\mathrm{Xi}=3$ and so on.

Cardiac related factors:

\begin{tabular}{cc}
\hline \multicolumn{1}{c}{ Risk factors } & Beta co-efficient value for \\
EuroSCORE II
\end{tabular}

Operation related factors:

\begin{tabular}{lc}
\hline Risk factors & Beta co-efficient value for EuroSCORE II \\
\hline Urgency of operation & \\
Urgent & 0.3174673 \\
Emergency & 0.7039121 \\
Salvage & 1.362947 \\
Weight of the intervention & 0.0062118 \\
Single non CABG & 0.5521478 \\
2 procedure & 1.362947 \\
3 procedure & 0.6527205 \\
Surgery on thoracic aorta & \\
\hline
\end{tabular}

\section{Follow up of patient}

Patients were discharged at the $7^{\text {th }}$ postoperative day routinely unless any of them were complicated by any factor.

1) Those who were successfully discharged was contacted via mobile phone at the $31^{\text {st }}$ postoperative day whether any mortality event occur and record accordingly.
2) Those who were complicated by any factor and had postoperative hospital stay prolong were followed till their discharge or any mortality event and record accordingly.

\section{Surgical Techniques \& Anesthesia:}

All patients were received General Anesthesia according to standard anesthetic protocol. A uniform operative 
technique were used. Surgical correction of all patients included in the study were done through standard median sternotomy and using cardiopulmonary bypass (CPB). A standard CPB circuit were used. After completing operative procedure, Protamine sulphate (100:1 ratio) were used to reverse the heparin effect at completion of the surgical procedure. Per-operative Aortic Cross clamp time, ECCT were recorded.

\section{Data processing}

Data were collected using a preformed data collection sheet (questionnaire). Baseline information was collected from the patient after exploration of different complains, sign and symptoms. Data acquisition were performed by using EuroSCORE II and original EuroSCORE datasheet from patients file and later compiled in online calculator present in this site http://www.euroscore.org/calc.html. Data were analyzed by computer software Statistical Packages for Social Scientist (SPSS) for windows version 20. The results were presented in tables. Continuous variables were expressed as mean \pm standard deviation and discrete variables were summarized by percentages. The cohort was grouped into low, medium and high risk group according to EuroSCORE II, additive and logistic version of EuroSCORE model. The validity of the model were analyzed by its calibration (statistical precession) with Hosmer-lemeshow chi square test and discriminatory capacity (statistical capacity) with ROC curve. And association between observed and predicted mortality were assessed by comparing the observed and predicted mortality figure with $95 \%$ confidence interval using Pearson chi square test, $p$ value less than $<0.05$ considered significant.

\section{Results:}

This was a prospective cohort study conducted in the department of cardiac surgery, from July 2016 to June 2018 among the patients admitted for valve surgery. After fulfilling the inclusion and exclusion criteria a total of 356 patients were enrolled in this study.

Distribution of the patients by age shows majority of patients were within $4^{\text {th }}$ decade and was mean age $43.29 \pm 12.35$. In this study $49.4 \%$ patients were male and $50.6 \%$ were female.

Table I shows that out of 356 patients 31 had extra cardiac arteriopathy among them 03 mortality were seen. Fisher's Exact test demonstrates that association between extra cardiac arteriopathy and early mortality was statistically significant. Association between diabetes on insulin and early mortality was statistically significant. Other factors demonstrates no association with early mortality and statistically not significant.

Most of the patient (281 out of 356) in this study population were in NYHA class II and shows mortality of $1.8 \%$. Also 68 out of 356 patient were in class III and shows mortality of $7.4 \%$. Fisher's Exact test demonstrates that association between NYHA class and early mortality was statistically not significant. Similarly, most of the patient ( 218 out of 356) in this study group have good left ventricular ejection fraction and 3 patient died, 7 mortality seen among patients with moderate LV function. Fisher's Exact test demonstrates that association between Left ventricular ejection fraction and early mortality was statistically not significant. Cardiac risk factors like CSS class IV angina, recent $\mathrm{Ml}$ (Within 90 days), Pulmonary Hypertension and association between urgency of operation and early mortality was not statistically significant.

Table II shows shows association between weight of intervention and early mortality was statistically not significant.

Table III shows shows single valve surgery mortality $2.3 \%$ ( 7 out of 296) and double valve surgery mortality $5.7 \%$ (3

Table-I

Distribution of the patient's risk factors by early mortality

\begin{tabular}{lccc}
\hline Risk factors & \multicolumn{2}{c}{ Early mortality (within 30 days) } & p value ${ }^{\mathrm{a}}$ \\
\cline { 2 - 3 } & Yes & No & \\
\hline Extra Cardiac arteriopathy & $3(9.7)^{\mathrm{b}}$ & $28(90.3)$ & 0.047 \\
Poor mobility & $0(.0)$ & $13(100.0)$ & 0.999 \\
Previous cardiac surgery & $1(3.7)$ & $26(96.3)$ & 0.550 \\
Chronic lung disease & $5(3.1)$ & $154(96.9)$ & 0.757 \\
Active endocarditis & $0(.0)$ & $16(100.0)$ & 0.999 \\
Clinical preoperative state & $1(2.1)$ & $46(97.9)$ & 0.999 \\
Diabetes on insulin & $8(4.7)$ & $162(95.3)$ & 0.003 \\
\hline
\end{tabular}

${ }^{a}$ Fisher's Exact test was done to measure the level of significance. $P$ value $<0.05$ is considered significant. ${ }^{b}$ Figure within parentheses indicates in percentage. 
Table-II

Distribution of the patients Weight of intervention by early mortality

\begin{tabular}{lccc}
\hline Weight of intervention & \multicolumn{2}{c}{ Early mortality (within 30 days) } & P value \\
\cline { 2 - 3 } & Yes & No & \\
\hline Single non CABG & $7(2.4)^{\mathrm{b}}$ & $281(97.6)$ & \\
2 Procedures & $3(4.4)$ & $65(95.6)$ & 0.410 \\
\hline Total & $10(2.8)$ & $346(97.2)$ & \\
\hline
\end{tabular}

aFisher's Exact test was done to measure the level of significance, $\mathrm{P}$ value $<0.05$ is considered significant. ${ }^{\mathrm{b}}$ Figure within parentheses indicates in percentage.

Table-III

Distribution of the single or double valve surgery by early mortality

\begin{tabular}{llcc}
\hline Weight of intervention & \multicolumn{2}{c}{ Early mortality (within 30 days) } & p value $^{a}$ \\
\cline { 2 - 3 } & Yes & No & 0.174 \\
\hline Single valve (Aortic /Mitral) & $7(2.3)$ & $296(97.7)$ & \\
Double valve & $3(5.7)$ & $50(94.3)$ & \\
\hline Total & $10(2.8)$ & $346(97.2)$ & \\
\hline
\end{tabular}

aFisher's Exact test was done to measure the level of significance. $P$ value $<0.05$ is considered significant. ${ }^{b}$ Figure within parentheses indicates in percentage.

out of 50), association between single and double valve operation and early mortality was statistically not significant.

Table IV demonstrate that in low risk group 158 patients with no mortality, in moderate risk group 121 patients with mortality $5(4.1 \%)$ and in high risk group 77 patients with mortality $5(6.5 \%)$.

Table $\mathrm{V}$ demonstrate that in low risk group 15 patients with no mortality, in moderate risk group 218 patients with mortality $4(1.8 \%)$ and in high risk group 123 patients with mortality 6 (4.9\%).

Table VI demonstrate that in low risk group 27 patients with no mortality ,in moderate risk group 168 patients with mortality $3(1.8 \%)$ and in high risk group 161 patients with mortality 7 ( $4.3 \%)$.

\section{Validation of EuroSCORE II model on NHFH\&RI sample}

Model calibration means how precisely model can predict the outcome. Model calibration was analyzed by determining Hosmer-Lemeshow goodness of fit statistic in multiple regression analysis. The Hosmer-Lemeshow chi-aquare statistic ( $\mathrm{C}$ statistic) measures the differences between expected and observed outcomes in different risk groups. A well calibrated model gives corresponding $p$ value greater than 0.05 that means all the risk factors taken into consideration to predict mortality therefore can accurately predicts operative mortality.
Here on NHFH\&RI cohort applying EuroSCORE the predicted and observed mortality after HosmerLemeshow test $p$ value found $>0.05$ in all subgroups including overall cohort.Therefore all risk factors taken into consideration in the EuroSCORE II model can accurately predict early mortality on NHFH\&RI cohort.

Table VII shows association between EuroSCORE II predicted mortality and observed early mortality was statistically not significant. Therefore all risk factors taken into consideration in the EuroSCORE II model can accurately predict early mortality on NHF cohort.

Table VIII shows association between Additive EuroSCORE predicted mortality and observed early mortality was statistically not significant. Therefore all risk factors taken into consideration in the Additive EuroSCORE model can accurately predict early mortality on NHF cohort.

Table IX shows association between Logistic EuroSCORE predicted mortality and observed early mortality was statistically not significant .Therefore all risk factors taken into consideration in the Logistic EuroSCORE model can accurately predict early mortality on National Heart Foundation cohort.

Performance of the patients by EuroSCORE II, Additive EuroSCORE and Logistic EuroSCORE in mortality prediction and comparing with early mortality: 
Table-IV

Distribution of the patients EuroSCORE II by early mortality

\begin{tabular}{lccc}
\hline EuroSCORE II & \multicolumn{2}{l}{ Early mortality (within 30 days) } & Total \\
\cline { 2 - 4 } & Yes & No & \\
\hline Low $(0-2)$ & $0(0)$ & $158(98.8)$ & $158(100.0)$ \\
Moderate (2-5) & $5(4.1)$ & $116(96.7)$ & $121(100.0)$ \\
High $(>5)$ & $5(6.5)$ & $72(94.7)$ & $77(100.0)$ \\
\hline Total & $10(2.8)$ & $346(97.2)$ & $356(100.0)$ \\
\hline
\end{tabular}

${ }^{\mathrm{b}}$ Figure within parentheses indicates in percentage.

Table-V

Distribution of the patient's additive EuroSCORE by early mortality

\begin{tabular}{llcc}
\hline Additive EuroSCORE & \multicolumn{2}{l}{ Early mortality (within 30 days) } & Total \\
\cline { 2 - 4 } & Yes & No & $15(100.0)$ \\
\hline Low $(0-2)$ & $0(.0)$ & $15(100.0)$ & $218(100.0)$ \\
Moderate $(2-5)$ & $4(1.8)$ & $214(98.2)$ & $123(100.0)$ \\
High $(>5)$ & $6(4.9)$ & $117(95.1)$ & $356(100.0)$ \\
\hline Total & $10(2.8)$ & $346(97.2)$ & \\
\hline
\end{tabular}

${ }^{\mathrm{b}}$ Figure within parentheses indicates in percentage.

Table-VI

Distribution of the patients logistic EuroSCORE by early mortality

\begin{tabular}{llcc}
\hline Logistic EuroSCORE & \multicolumn{2}{c}{ Early mortality (within 30 days) } & Total \\
\cline { 2 - 4 } & Yes & No & $27(100.0)$ \\
\hline Low $(0-2)$ & $0(.0)$ & $27(100.0)$ & $168(100.0)$ \\
Moderate $(2-5)$ & $3(1.8)$ & $165(98.2)$ & $161(100.0)$ \\
High $(>5)$ & $7(4.3)$ & $154(95.7)$ & $356(100.0)$ \\
\hline Total & $10(2.8)$ & $346(97.2)$ & \\
\hline
\end{tabular}

${ }^{\mathrm{b}}$ Figure within parentheses indicates in percentage.

Table-VII

Calibration of the EuroSCORE II model on overall patients

\begin{tabular}{|c|c|c|c|c|}
\hline \multirow[t]{2}{*}{ Category } & \multirow[t]{2}{*}{ No. of Patients } & \multicolumn{2}{|c|}{ Early mortality } & \multirow[t]{2}{*}{$p$ value ${ }^{\star}$} \\
\hline & & Observed & Expected & \\
\hline 1 & 38 & 0 & 0.625 & \\
\hline 2 & 37 & 0 & 0.632 & \\
\hline 3 & 37 & 0 & 0.654 & \\
\hline 4 & 37 & 0 & 0.691 & \\
\hline 5 & 35 & 0 & 0.704 & 0.226 \\
\hline 6 & 36 & 2 & 0.781 & \\
\hline 7 & 36 & 2 & 0.901 & \\
\hline 8 & 36 & 2 & 1.109 & \\
\hline 9 & 36 & 3 & 1.474 & \\
\hline 10 & 28 & 1 & 2.430 & \\
\hline
\end{tabular}

*Hosmer and Lemeshow test was done to measure the level of significant, $\mathrm{P}$ value $<0.05$ is considered significant. 
Table-VIII

Calibration of the Additive EuroSCORE model on overall patients

\begin{tabular}{lcccc}
\hline Category & No. of Patients & \multicolumn{2}{c}{ Early mortality } & p value \\
\cline { 2 - 4 } & & Observed & Expected & \\
\hline 1 & 15 & 0 & 0.222 & \\
2 & 96 & 0 & 1.713 & \\
3 & 65 & 1 & 1.398 & 0.221 \\
4 & 57 & 3 & 1.476 & \\
5 & 49 & 3 & 1.527 & \\
6 & 29 & 2 & 1.086 & \\
7 & 45 & 1 & 2.578 & \\
\hline
\end{tabular}

*Hosmer and Lemeshow test was done to measure the level of significant, $\mathrm{P}$ value $<0.05$ is considered significant.

Table-IX

Calibration of the Logistic EuroSCORE model on overall patients

\begin{tabular}{lcccc}
\hline Category & No. of Patients & \multicolumn{2}{c}{ Early mortality } & p value \\
\cline { 2 - 4 } & & Observed & Expected & \\
\hline 1 & 27 & 0 & 0.505 & \\
2 & 35 & 0 & 0.683 & \\
3 & 36 & 0 & 0.721 & \\
4 & 37 & 1 & 0.787 & 0.641 \\
5 & 27 & 0 & 0.594 & \\
6 & 33 & 2 & 0.772 & \\
7 & 36 & 2 & 0.894 & \\
8 & 36 & 1 & 1.017 & \\
9 & 36 & 1 & 1.207 & \\
10 & 53 & 3 & 2.821 & \\
\hline
\end{tabular}

*Hosmer and Lemeshow test was done to measure the level of significance, $\mathrm{P}$ value $<0.05$ is considered significant.

Table $X$ shows association between observed early mortality and predicted mortality by EuroSCORE II, Additive EuroSCORE and Logistic EuroSCORE was statistically not significant.

\section{Discriminatory capacity of the EuroSCORE model}

It was analyzed by calculating the area under the ROC curve. The models ability to discriminate was assessed in terms of its capacity to distinguish between patients who died during hospitalization from those who did not. Typical $\mathrm{C}$ values for cardiac surgery models range from 0.72 to 0.76 that means prediction of individual outcome is correct $72 \%$ to $46 \%$ of the time. A value of 0.5 indicates no discrimination and a value of 1.0 indicates perfect predictor. Areas of greater than 0.7 are generally thought to be useful. The discriminatory power of the model is Excellent if ROC exceeds 0.80 , Very good if it exceeds 0.75 and good if it exceeds 0.70 .
In this study, the model's discriminatory power was found good and useful, as indicated by an area under ROC curve of 0.779 in EuroSCORE II model, 0.675 in additive method and 0.696 in logistic method that means EuroSCORE II can predict the outcome with $77 \%$ accuracy, additive method does with $67 \%$ accuracy and logistic method with $69 \%$ accuracy.

The Table XI demonstrates that EuroSCORE II bears area under Receiver Operating Characteristic (ROC) curve 0.779 which means it can predict mortality with $77 \%$ accuracy, whereas AUC of ROC curve of additive EurOSCORE and logistic EurOSCORE was 0.675 and 0.696 respectively. EuroSCORE II and Logistic EuroSCORE shows $p$ value $<0.05$ which indicates result statistically significant. Additive EuroSCORE shows $P$ value $>0.05$ which indicates result statistically not significant. 
Table-X

Association between predicted mortality and observed early mortality

\begin{tabular}{lcccc}
\hline EuroSCORE & $\begin{array}{c}\text { Observed early } \\
\text { mortality (\%) }\end{array}$ & $\begin{array}{c}\text { Predicted } \\
\text { mortality (\%) }\end{array}$ & $95 \% \mathrm{Cl}$ & $\mathrm{p}$ value \\
\hline EuroSCORE II & 2.8 & 2.73 & $1.02-4.38$ & 0.884 \\
Additive EuroSCORE & 2.8 & 2.15 & $0.68-3.72$ & 0.786 \\
Logistic EuroSCORE & 2.8 & 2.25 & $0.74-3.86$ & 0.852 \\
\hline
\end{tabular}

${ }^{*}$ One proportion $\mathrm{z}$ test was done to measure the level of significance, $\mathrm{P}$ value $<0.05$ is considered significant. \#Null hypothesis is observed mortality. The performance of the model was assessed by comparing the observed and predicted figures with $95 \%$ confidence intervals.

Table-XI

Area Under the ROC Curve of EuroSCORE II, Additive EuroSCORE and Logistic EuroSCORE

\begin{tabular}{|c|c|c|c|c|}
\hline \multirow[t]{2}{*}{ Test Result Variables } & \multirow[t]{2}{*}{ Area under the ROC Curve } & \multirow[t]{2}{*}{$p$ value ${ }^{\#}$} & \multicolumn{2}{|c|}{ 95\% Confidence Interval } \\
\hline & & & Lower Bound & Upper Bound \\
\hline EutoSCORE II & 0.779 & 0.003 & 0.690 & 0.867 \\
\hline Additive EuroSCORE & 0.675 & 0.059 & 0.543 & 0.807 \\
\hline Logistic EuroSCORE & 0.696 & 0.034 & 0.594 & 0.799 \\
\hline
\end{tabular}

\#Null hypothesis: true area $=0.5, \mathrm{P}$ value $<0.05$ is considered significant.

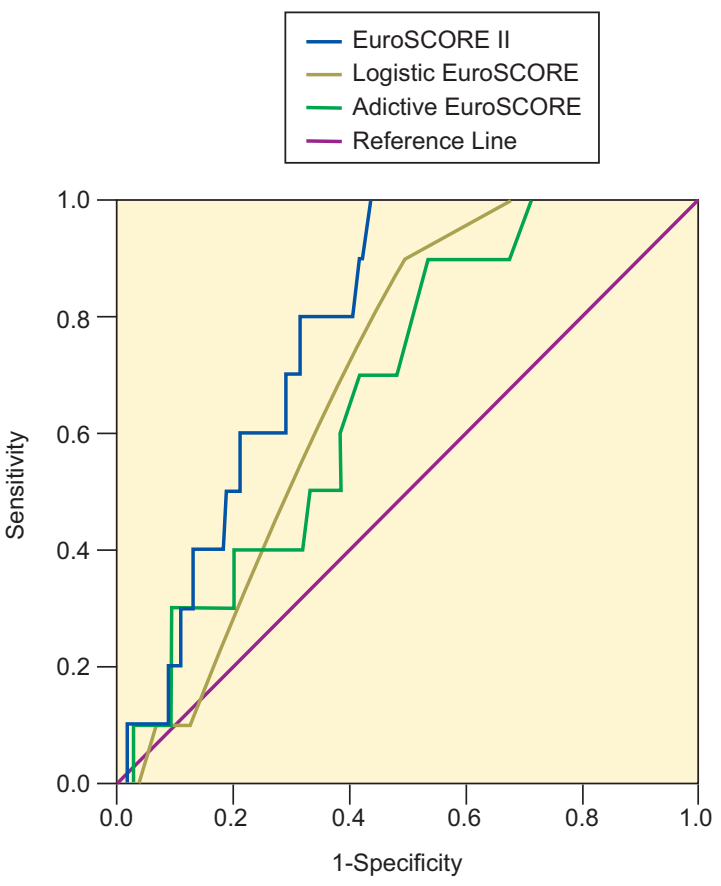

Fig.-1: ROC curve of EutoSCORE II, Additive EuroSCORE and Logistic EuroSCORE.

\section{Discussion:}

The current clinical profile of patients submitted to heart surgery puts into question the incidence of operative or hospital deaths as reliable indicators and not sufficient to evaluate the quality of services provided by the cardiac hospital. Without individual risk adjustment, taking into considerations associated risk factors, the conclusions might be incorrect. Prognostic models which take into account patients specific characteristics and which provides risk adjusted outcomes for intervention are required and more useful to allow meaningful comparison of outcomes to be performed between institutions and surgeons.

The choices available to a surgical unit are to create a new model, recalibrate an existing model or to use a ready-made model. The former two options are impractical to most cardiac surgical units as they require comprehensive database management and accumulation of large patient number to allow creation or recalibration of a model .The use of a ready-made widely used model has the additional advantage of allowing meaningful comparison to be made regionally and internationally.

Different systems of risk stratification have been utilized over the last two decades in different countries with the objective of predicting mortality in cardiac surgery. Additionally the application of a scoring system provides information to patients and their families in respect to the risk that individual patients will be submitted.

In the recent years the EuroSCORE has become one of the most widely used prognostic scoring system. Among the different studies to stratify the risk, the Euro score 
has proved to be accurate, even when applied to nonEuropean populations. Some countries found poor calibration of this model on their population and therefore they readjusted that version of EuroSCORE on their population. EuroSCORE is easy to use at the bedside and allows an analysis very close to the results, particularly in surgeries of the valve surgeries.

National heart foundation hospital \& research institute is a reference centre for this type of heart surgery and performs a large numbers of valve surgeries every year. Before using a probabilistic model in a context other than upon which it was created should be validated otherwise it may produce erroneous probabilities.

From January 2008 to December 2009 a prospective cohort study was done among 234 patients who underwent coronary artery bypass grafting in this institute to validate the former EuroSCORE model. Calibration was analysed by Hosmer-Lemeshow goodness of fit test. The area under ROC curve in additive method was found 0.74 and 0.73 in logistic method. In that study both the additive and logistic method of EuroSCORE showed good calibration and discrimination (Rahman, et al., 2012)

The EuroSCORE was recently reported to overestimates the cardiac surgery risk because of improved outcomes in cardiac surgery and marked reduction of risk adjusted mortality. To overcome this limitation, an updated version of this model named EuroSCORE II was developed. The purpose of this study was to assess the performance of the EuroSCORE II and to compare its prediction performance with that of original model.

We prospectively applied the EuroSCORE to analyse early mortality in 356 consecutive patients who underwent elective valve surgery in NHFH. In the original work (Nashef, et al., 2012) studied on 22,381 patients in 154 hospitals in 43 countries (average 145 patients per centre). Many risk factors were critically observed in this sample. The mean age of our sample was $43.29 \pm 12.35$ and of the EuroSCORE II was $64.6 \pm 12.5$.in our country there is no patient over 72 years.

In our patient majority were female $50.6 \%$ ( 180 out of 356 ) and male were $49.4 \%$ (176 out of 356 ). According to Nashef, et al., females have a higher mortality than males.

The mean serum creatinine of our study was $1.10 \pm 0.21$ $t$ test was done to measure the level of significance $P=$ 0.927 and it is considered not significant. Renal function was assessed by the estimated creatinine clearance using the Cockroft-Gault formula, and it is a better predictor than serum creatinine (Nashef,et al.,2012). In this study early mortality is seen in moderately impaired renal function.
This study shows the significant relation between extra cardiac arteriopathy, diabetes on insulin and early mortality. Fisher's Exact test was done to measure the level of significance which shows $p$ value 0.047 and 0.053 respectively. Poor mobility, chronic lung disease, previous cardiac surgery, critical preoperative state, active endocarditis shows non-significant on Fisher's Exact test.

Among the cardiac factors, left ventricular ejection fraction ( LVEF) of major portion of our patient of this study had good left ventricular ejection fraction, only 3 patient had poor ejection fraction and no mortality was seen in this group .Patients with moderate ejection fraction shows mortality of $5.2 \%$ ( 7 out of 135). Fisher's Exact test was done to measure between other factors and early mortality CCS class IV angina, recent MI, and pulmonary hypertension shows $p$ value of $0.999,0.999$ and 0.999 respectively and all of them considered non-significant.

Among the operation related factors, weight of intervention shows no significant association with early mortality. Chisquare test was done to measure the level of significance which shows $p$ value of 0.410 (considered not significant)

In this study group distribution of early mortality in EuroSCORE II Mean \pm SD (4.6 \pm 3.19$)$, Mann-Whitney U test to measure the level of significance showed $p$ value of 0.003 ( considered significant), whereas distribution of additive EuroSCORE $(6.00 \pm 1.41)$ with $p$ value 0.059 (considered not significant); distribution of logistic EuroSCORE $(8.21 \pm 5.03)$ with $p$ value 0.034 (considered significant).

Study population were grouped into three category, in EuroSCORE II $45 \%$ patients falls into low risk, $33.7 \%$ in moderate risk and $21.3 \%$ in high risk group. In additive EuroSCORE $4.2 \%$ patient in low risk group followed by moderate risk $61.2 \%$ and high risk $34.6 \%$. Where in logistic EuroSCORE $7.5 \%$ patient in low risk group followed by $47.3 \%$ moderate risk and $45.2 \%$ in high risk group.

In this study, the $\mathrm{C}$ statistics obtained with the HosmerLemeshow test was $p=0.226$ in EuroSCORE II, $p=0.221$ in additive method and $p=0.641$ in logistic method and were not significant in different risk groups of $\mathrm{NHF}$ sample and $p$ value remains $>0.05$ in all subgroups which indicates risk factors used EuroSCORE model could predict the operative mortality satisfactorily for patients undergoing valve surgery in our centre.

The area under ROC curve was 0.8905 in the original EuroSCORE II data set (Nashef, et al., 2012) In this study, the model's discriminatory power also good and useful, as indicated by an area under ROC curve of 0.779 in EuroSCORE II model, 0.675 in additive method and 0.696 
in logistic method that means EuroSCORE II method can predict the outcome with $77 \%$ accuracy, additive method with $67 \%$ accuracy and the logistic method does that with $69 \%$ accuracy. It signifies that EuroSCORE II model has better discriminatory power than the additive and logistic version of EuroSCORE.

The overall mortality in our setting was $2.80 \%$ (10 out of $356)$ and predicted mortality was $2.73 \%$ (95\% confidence interval 1.02-4.38) by the EuroSCORE II, 2.15\% (95\% confidence interval 0.68-3.72) by the additive method and $2.25 \%$ (95\% confidence interval $0.74-3.86$ ) by the logistic EuroSCORE.

One proportion $Z$ test was done to see the association between the predicted mortality using EuroSCORE II, additive and logistic method with the observed early mortality. The performance of the model was assessed by comparing the observed and predicted mortality figures with $95 \%$ confidence intervals. The observed early mortality in three different risk groups was compared by univariate analysis with predicted mortality by EuroSCORE II, additive and logistic method of EuroSCORE. The $p$ value was 0.884 in EuroSCORE II, 0.786 in additive method and 0.852 in logistic method of EuroSCORE that means both EuroSCORE II predicted mortality and mortality predicted by logistic EuroSCORE is as similar to observed early mortality for valve surgery in National Heart Foundation Hospital \& Research Institute.

By this present study, we validated the EuroSCORE II model for use in this centre and that it has been proven to be a reliable instrument for risk stratification. This signifies that the model's predictions of the probability of dying are valid and appropriately risk-adjusted for patients undergoing valve surgery in National Heart Foundation Hospital \& Research Institute.

Limitation: This analysis was done in a single centre of Bangladesh, and the sample represents only a fraction of patients undergoing valve surgery in Bangladesh. The focus of this study was adult patients undergoing valve surgery, the identified independent risk factors may not be applicable to other surgeries as aortic surgery, congenital heart surgery or heart transplantation.

\section{Conclusion:}

The results of this study allow us to conclude that despite substantial demographic and epidemiological differences between Bangladesh and European population EuroSCORE II was validated and performed well on NHFH\&RI patients and could be recommended as a simple risk stratification system to estimate the probability of early mortality in patients scheduled for valve surgery in Bangladesh.
This study demonstrates that the EuroSCORE II is more accurate in predicting operative mortality than the additive EuroSCORE (AES) and logistic EuroSCORE (LES) in patients undergoing valve surgery patients. The model validated in the present study could be useful in providing systematic information on the outcome of valve surgeries in other centers of Bangladesh.

\section{References:}

1. Alessandro, P., Lorenzo, L.P., Matteo, T., Laura, C., Samer, K., Claudia, L., Davide, P., Elena, T. and Francesco, A., 2010. EuroSCORE Performance in Valve Surgery: A Meta-Analysis. Annals of Thoracic Surgery, 89, pp.787-793.

2. Ayse, G.K., Murat, K., Mete, H., Levent, C., Aslihan, K., Vedat, B., Ahmet, R.A. and Erol, S., 2013.Comparison of original EuroSCORE, EuroSCORE II and STS risk models in a Turkish cardiac surgical cohort. Interactive Cardiovascular and Thoracic Surgery, 16, pp. 625-629.

3. Alexander, S., Jan-Malte, S., Mariuca, Vasa-N., Alexander, G., Christoph, H., Eberhard, G., Georg, N. and Nikos, W., 2013. The revised EuroSCORE II for the prediction of mortality in patients undergoing transcatheter aortic valve implantation. Clinical Research in Cardiology, 10, pp.596-598.

4. Dimitri, A., Claire, C., Soleiman, A., Andrea, C., Dominique, H., Eric, B., Bernard, I., Patrick, N., JeanPol, D., Alec, V. and David, M., 2014. Agreement between the new EuroSCORE II, the Logistic EuroSCORE and the Society of Thoracic Surgeons score: Implications for transcatheter aortic valve implantation. Archives of Cardiovascular Disease, 107, pp.353-360.

5. Domenico, P., Pietro, G., Giuseppe, D.E., Sergio, C., Renato, G., Mauro, C., Vitantonio, F., Giuseppe, S., Valerio, M., Salvatore, Z., Luigi, D.L.T.S. and Tommaso, F., 2014. Risk stratification for in-hospital mortality after cardiac surgery: external validation of EuroSCORE II in a prospective regional registry. European Journal of Cardio-Thoracic Surgery, [ejournal] pp.1-9. http://doi.org /10.1093/ejcts/ezt657

6. Fabio, B., Davide, P., Mariangela, D., Nicholas, C.D., Francesco, A., Roberto, D.B., Claudio, G., Marina, D., Danilo, F. and Alessandro, P., 2016 .The Impact of EuroSCORE II Risk Factors on Prediction of LongTerm Mortality. Annals of Thoracic Surgery, 102, pp.1296-1303.

7. Guan, Z., Chong, W., Wang, L., Fang, L., Bai-ling, L., Lin, H. and Zhi-yun, X., 2013. Validation of 
EuroSCORE II in Chinese Patients Undergoing Heart Valve Surgery. Heart, Lung and Circulation, 22, pp.606-611.

8. Hernandez, V.D., Rocio, D., Ruben, A.C., Carlos, M. and Jacob., 2015. Performance of EuroSCORE II in octogenarians with comorbidities undergoing aortic valve replacement. Journal of Cardiothoracic Surgery, 10, p.57.

9. Ioannis, K.T., Constantine, E.A., Stavros, K.T., Joseph, J.D. and Daniel, G.S., 2005. EuroSCORE Predicts Long-Term Mortality After Heart Valve Surgery. Annals of Thoracic Surgery, 79, pp.1902-1908.

10. Isaac, N.G.A., Fernando, R.D.M., Joao, P.S.D.P., Igor, T.C., Tamyris, G. and Carlos, R.R., 2010. Assessment of the EuroSCORE as a predictor for mortality in valve cardiac surgery at the Heart Institute of Pernambuco .The Brazilian journal of Cardiovascular surgery, 25(1), pp. 11-18.

11. Johan, N., Lars, A., Peter, H.G., Carsten, L. and Johan, B., 2004. EuroSCORE Predicts Intensive Care Unit Stay and Costs of Open Heart Surgery. Annals of Thoracic Surgery, 78, pp.1528 -1535.

12. Wang, L., Qing-Qi, H., Fan, Q., Chong, W., Xi-Wu, Z., Lin, H. and Zhi-Yun, X., 2013. Performance of EuroSCORE II in patients who have undergone heart valve surgery: a multicenter study in a Chinese population. European Journal of Cardio-Thoracic Surgery, 10, pp. 1-6.

13. Mohamed, A.A. and Ashraf, A.M.E.S., 2016. Evaluation of accuracy of EuroSCORE II in prediction of in-hospital mortality in patients underwent valve replacement in Egypt. Journal of the Egyptian Society of Cardio-Thoracic Surgery, 24, pp. 135-142.

14. Manuel, C., Jacobo, A.S.G., Fernando, J.R.L., Luis, C.M.C., Javier, C., Enrique, V.M., Teresa, T.S. and José, E.R.H., 2013. Validation of EuroSCORE II on a single centre 3800 patient cohort. Interactive CardioVascular and Thoracic Surgery, 16, pp. 293-300.

15. Michael, P., 2014. Recursive and non-linear logistic regression: moving on from the original EuroSCORE and EuroSCORE II methodologies. Interactive CardioVascular and Thoracic Surgery, 19, pp. 726-734.

16. Muhammad, S.R., Irfan, Q., Yasir, A., Marrium, G. and Hasanat, Sharif., 2014. Heart valve surgery: EuroSCORE vs. EuroSCORE II vs. Society of
Thoracic Surgeons score. Heart International, 9 (2), pp. 53-58.

17. Nashef, S.A., Roques, F., Sharples, L.D., Nilsson, J., Smith, C. and Goldstone, A.R., 2012. EuroSCORE II. European journal of cardiothoracic surgery, 41, pp.734-744.

18. Nirmal, K., Prashant, S., Subodh, S., Saket, A., Vithal, K.B. and Deepak, K.S., 2013. Early Results of Mitral Valve Replacement in Severe Pulmonary Artery Hypertension- an Institutional Prospective Study. World Journal of Cardiovascular Surgery, 3, pp. 6369.

19. Niv, A., Sari, D.H., Jay, P., Graciela, P., Deborah, J.S. and Linda, H., 2016. Comparison of EuroSCORE II, Original EuroSCORE, and The Society of Thoracic Surgeons Risk Score in Cardiac Surgery Patients. Annals of Thoracic Surgery, 102, pp.573-579.

20. Rahman, M.Z., Sayeed, S., Choudhury, S.R., Rasheed, H., Sharifuzzaman, M. and Ahmed, F., 2012. Single center performance of the EuroSCORE on a consecutive sample of coronary artery bypass surgery in a developing country. Journal of National Heart Foundation Hospital Bangladesh, 1, pp.58-61.

21. Roques, F., Nashef, S.A., Michel, P., Gauducheau, C., Vincentiis, D., E. Baudet, J., Cortina, M., David, A.F., Gabrielle, F., Gams, E., Harjula, A., Jones, M.T., Pinna, P., Salamon, R. and Thulin, L., 1999. Risk factors and outcome in European cardiac surgery: analysis of the EuroSCORE multinational database of 19030 patients. European Journal of Cardiothoracic Surgery, 15, pp.816- 823

22. Raúl, A.B., Miguel, R., Leonardo, C., Carlos, A.I., Norberto, G.A. and Rodolfo, A.A.G., 2014. Prospective validation of EuroSCORE II in patients undergoing cardiac surgery in Argentinean centres. Interactive CardioVascular and Thoracic Surgery, 18, pp. 539-543.

23. Ricardo, C., Flávio, T., Guilherme, S., Marcelo, K., Antonio, B., Roney, S., Otavio, T.R., Pablo, M.P. and Max, G., 2015. EuroSCORE Models in a Cohort of Patients with Valvular Heart Disease and a High Prevalence of Rheumatic Fever Submitted to Surgical Procedures. PLOS ONE, [e-journal] 10, pp.1371. http://doi.or g/10.1371/journal.pone. 0118357 
24. Roques, F., Micheal, P., Goldstone, A.R. and Nashef, S.A., 2003. The logistic EuroSCORE. European Heart Journal, 24, pp.1-2. http://doi.org/10.1016/ S0195-668.(02)00799-6

25. Samer, A.M.N., Francois, R., Linda, D.S., Johan, N., Christopher, S., Antony, R.G. and Ulf, L., 2012. EuroSCORE II. European Journal of CardioThoracic Surgery, 41, pp. 734-745.

26. Shahab, N., Johan, S., Torbjorn, I., Peter, H. and Johan, Nilsson., 2011. Validation of a modified EuroSCORE risk stratification model for cardiac surgery: the Swedish experience. European Journal of Cardio-thoracic Surgery, 40, pp.185-191.

27. Stavridis, G., Panaretos, D., Kadda, O. and Panagiotakos, D.B., 2017. Validation of the EuroSCORE II in a Greek Cardiac Surgical Population: A Prospective Study. The Open Cardiovascular Medicine Journal, 11, pp. 94-101.

28. Shanmugan, G., West, M. and Berg, G., 2005.Additive and logistic EuroSCORE performance in high risk patients. Interactive Cardiovascular and Thoracic Surgery, 4, pp.299-303.

29. Sophie, P., Arnaud, C., Walid, G., Claire, B., Philippe, M., Dan, L. and Bernard, I., 2017. Is the EuroSCORE
II reliable to estimate operative mortality among octogenarians? PLOS ONE, [e-journal] 12, pp.11. https://doi.org/10.1371/journal.pone.0187056

30. Takahiro, N., Hiromichi, S., Yasuhisa, O., Yoshihisa, T., Atsuhiro, N., Yuichi, S. and Ryuji, T., 2014. The novel EuroSCORE II algorithm predicts the hospital mortality of thoracic aortic surgery in 461 consecutive Japanese patients better than both the original additive and logistic EuroSCORE algorithms. Interactive Cardiovascular and Thoracic Surgery, 18, pp. 446-450.

31. Themistocles, E., Efstratia, C., Panagiotis, D., Christos, C. and Christina, R., 2015. Scoring Systems for Outcome Prediction in a Cardiac Surgical Intensive Care Unit: A Comparative Study. American Journal of Critical Care, 24, pp. 4.

32. Vincent, C., Azin, A., Marc, R., Elsayed, E., Mark, H. and Thierry, G.M., 2014. Perioperative Deaths After Mitral Valve Operations May Be Overestimated by Contemporary Risk Models. Annals of Thoracic Surgery, 98, pp.605-610.

33. EuroSCORE interactive calculator, 2011.http:// www.euroscore.org/calcold.html. 